\title{
Radiological resolution of nutritional rickets with mega dose of vitamin D - "Stoss Therapy": a hospital-based study
}

\author{
Kumar M. ${ }^{1}$, Pheroz M. ${ }^{2}$, Gupta V. ${ }^{3}$, Pandey V.K. ${ }^{4}$, Jain A. ${ }^{5}$, Sharma V. ${ }^{6}$ \\ ${ }^{1}$ Dr. Manoj Kumar, ${ }^{2}$ Dr. Mozammil Pheroz, Senior Resident, ${ }^{3}$ Dr. Vikas Gupta, Professor, Safdarjung Hospital, New \\ Delhi, India, ${ }^{4}$ Dr. Vikash Kumar Pandey, ${ }^{5}$ Dr. Ankit Jain, 1,2,4,5authors are Senior Resident, CIO, Safdarjung Hospital, \\ New Delhi, India, ${ }^{6}$ Dr. Vasu Sharma, Consultant, Orthopaedics Surgeon, Sita Ram Bharti Institute of Science and \\ Research, New Delhi, India
}

Corresponding Author: Dr. Mozammil Pheroz, Room No 63, Resident Doctor Hostel, Safdarjung Hospital, New Delhi110029, India. E-mail: muzammilphrz@gmail.com

\begin{abstract}
Background: Nutritional rickets is one of the most common metabolic disease of bone. The purpose of this study is to evaluate the level of Vitamin-D in children with rickets and establish that rickets in India is due to Vitamin-D deficiency and to compare and analysed the pre- and post-treatment radiographic parameters in nutritional rickets being treated by Stoss therapy. Interventional study, done at Central Institute of Orthopaedics, Safdarjung Hospital, New Delhi, India between October 2015 and May 2017. Material and Methods: A total of 44 patients in the age group of 6 months to 16 years with signs of active rickets participated in the study. All the participants received Stoss therapy (mega dose of vitamin D) and the response to treatment was observed and recorded at regular follow up with clinical, biochemical and radiological parameters. Thacher's score was used for radiological evaluation. Results: After starting treatment, 4 subjects out of 44 had zero score at 3 weeks. whereas at 6 weeks, 3 month 10 subjects and 17 subjects had score zero respectively. At 6 month all subject had score zero. Conclusion: The Stoss therapy is very effective and safe regimen for the treatment of rickets. The 10 points Thacher's scoring method for assessing the severity of rickets shows promise as a useful tool for researchers and clinicians.
\end{abstract}

Keywords: Nutritional Rickets, Stoss Therapy, Thacher's Score, Radiological resolution

\section{Introduction}

Rickets, a metabolic bone disease, characterized by defective mineralization of newly formed bone matrix is caused by Vitamin D deficiency and/or dietary calcium before epiphyseal fusion [1].

Nutritional rickets has rapidly gained public health attention worldwide during the past decade owing to a recent surge of cases, especially in developed countries, where it was thought to have been eradicated and contributes to childhood disability and morbidity $[2,3]$.

Rickets has been ranked among the five most prevalent diseases among children in developing countries [4].

The diagnosis of rickets is based on clinical features, biochemical studies and radiological signs and confirmed by response to treatment [5].

Manuscript Received: $23^{\text {rd }}$ September 2019

Reviewed: $2^{\text {nd }}$ October 2019

Author Corrected: $7^{\text {th }}$ October 2019

Accepted for Publication: $12^{\text {th }}$ October 2019
Radiographic changes of active rickets are evident at growth plate of rapidly growing long bones. These changes reflect expansion of the cartilaginous growth plate and delayed mineralization. The junction of the mineralizing metaphysis with the cartilaginous physis (zone of provisional calcification) increase in longitudinal thickness. Consequently, the lucent gap between metaphysis and epiphysis expands because the shadow of the zone of provisional calcification is partially or totally invisible [6].

The changes occurring in metaphysis may appear to be indistinct, frayed, and irregular on $\mathrm{x}$ ray examinations [7].

The visible calcified margin of the metaphysis can be concave and spread transversely, resulting in a cupped shape. Other radiographic sign of rickets includes generalized osteopenia, green-stick fracture, delayed appearance of ossification centers, and curving of long 


\section{Original Research Article}

bone. Though changes of rickets on radiographs are well characterized, there is no specific method for grading severity of these changes has been in general use. Consequently, it is difficult to compare objectively or follow radiographic improvement. To our knowledge, only one study by Thachers.et.al [8] conducted to evaluate the utility and reproducibility of a 10 -points scoring method for measuring the severity of rickets.

The purpose of this study is to evaluate the level of Vitamin-D in children with rickets and establish that rickets in India is due to Vitamin-D deficiency and to compare and analyse the pre- and post- treatment radiographic parameters in nutritional rickets being treated by Stoss therapy (single mega dose of vitamin D) with help of Thacher's scoring technique so to determine its usefulness as a tool for monitoring the improvement and formulate a reliable, accurate, yet cost-effective follow up regime that can be useful in less developed countries where rickets is endemic.

\section{Materials and Methods}

This was a longitudinal prospective study conducted between October 2015 and May 2017. Total number of 44 patients participated in the study.

Inclusion criteria: Only the patients aged 6 months to 16 years presenting at Safdarjung hospital were included in the study. Other criteria were evident clinical, biochemical and radiological changes of rickets, no previous treatment with vitamin $\mathrm{D}$ or calcium.

Exclusion criteria: Any patient with history of premature birth, any hepato-renal or intestinal malabsorption syndrome, any chronic disease in the patient like tuberculosis, vitamin D resistant rickets, any benign or malignant tumour patient were all excluded from the study. Informed consent was obtained from each patient's attendant. 44 presenting with clinical features of lower limb deformity (genu varum and valgum), 42 presenting with wrist and ankle joint swelling, a significant limp, or non-traumatic joint pain were screened for nutritional rickets. Each child underwent physical examination. A detailed dietary history was elicited. Family size, number of earning members, number and gender of children, preference for vegetarian food, composition of meals, and duration of breastfeeding and the amount of milk consumed in a day and sunlight exposure in min/week were recorded. Radiographs of wrists, knees, ankles were evaluated and scoring according to Thacher's 10-points scoring system given. The serum 25-OH Vitamin D, calcium, phosphate, and alkaline phosphatase levels, Liver Function Test, Kidney Function Test, Albumin were estimated. The diagnosis of nutritional rickets was made based on a combination of clinical (exclusion of other causes), radiological (widening of the physis, metaphyseal strips and in severe cases the typical cupping, splaying and fraying of the metaphysis, as well as decreased metaphyseal bone density) and biochemical (low serum calcium and phosphorus and raised alkaline phosphatase levels) criteria, and confirmed by responses to treatment.

Patients with clinical features suggestive of history of prematurity, any concurrent systemic illness, intake of any drug interfering with bone turnover, arthropathies, and any other skeletal disease, Tuberculosis, vitamin D resistant rickets, benign and malignant tumor were excluded. Patients with rickets caused by chronic diarrhea, renal or hepatic disease, malabsorption, epilepsy, and fluorosis were also excluded based on history and clinical examination of various systems, as well as investigation of haematological, urine, stool and imaging results.

Intervention: Children with nutritional rickets were treated by a single large intramuscular dose of vitamin D (600 000 IU) [9] along with oral calcium $(50 \mathrm{mg} / \mathrm{kg} /$ day) and supplementary vitamin D (400 IU/day) as well as advice on diet and sunlight exposure.

Outcome measures and scores used: Thacher's radiological scoring system was used for radiological resolution evaluation. Every Patient was followed up at 3 weeks to assess the responses to therapy and then at a 6 weeks interval until radiological and biochemical resolution occurs. Subsequent evaluation carried out at 12 week, $6^{\text {th }}$ month, $9^{\text {th }}$ month and 1 year and result were monitored clinically by determining any improvement in the clinical features, radiologically by Thacher's radiological scoring system and biochemically by estimation of serum calcium, phosphate, alkaline phosphatase and 25-OH Vitamin D.

Statistical analysis: Improvement in clinical, radiological, biochemical parameters were evaluated statistically with Chi-square test for qualitative and paired $\mathrm{t}$-test for quantitative data respectively. A $\mathrm{p}$ value of $>0.05$ was considered statistically significant. The data was entered in MS EXCEL spreadsheet and analysis was done using Statistical Package for Social Sciences (SPSS) version 21.0. This study was approved by the ethical committee of the hospital. 


\section{Results}

44 patients (25 males and 19 females) with nutritional rickets met the selection criteria and were enrolled in the study. The mean age of presentation was 4 years (range, 6 months to 12 years).73\% of subjects having sunlight exposure of less than 30 min per week. Those who were exposed to sunlight were partially dressed during the exposure. A history of prolonged breast feeding for more than 6 months was found in all the cases, with a mean of 1 year 8 months (range, 8-42 months).

Swollen wrist and ankle was the most common presentation being present in $95.5 \%$ followed by genu varum, frontal bossing, weight $<3^{\text {rd }}$ percentile, pot belly, height $<3^{\text {rd }}$ percentile, genu valgum, dental hypoplasia, rickety rosary respectively. Craniotabes, delayed fontanelle closure, kyphoscoliosis, raised intracranial pressure and delayed milestones had the least common presentation and were found in none of the study subjects.

In the present study it was noted that the mean radiological score at initial presentation as per Thacher's radiological scoring system was 6.9 with 8 out of 44 study subjects having a score of 10 and none having a score of 0 . The mean score at 3 weeks was 4.9 with $4(9.09 \%)$ study subjects having a score of 0 . The mean score at 6 weeks was 2.6 with 10 $(22.72 \%)$ study subjects having a score of 0 . The mean score at 3 months was 1.3 with 17 (33.63\%) study subjects having score 0. By 6 months, all the study subjects had complete radiological resolution (Table 1). All the changes of radiological scores during the follow up were found to be statistically significant by paired t test (Table 3 ). Time for the radiological score to be 0 had a mean of 129.5 days (approximately 4 months) ranging from 57 to 185 days.

Table-1: Radiological score distribution.

\begin{tabular}{|l|c|c|c|c|c|c|c|}
\hline \multicolumn{2}{|l|}{ Radiological score distribution } \\
\hline $\mathbf{n = 4 4}$ & 0 week & 3 weeks & 6 weeks & 3 months & 6 months & 9 months & 12 months \\
\hline Mean & 6.9 & 4.9 & 2.6 & 1.3 & 0 & 0 & 0 \\
\hline $\begin{array}{l}\text { No. of patients } \\
\text { having zero } \\
\text { score }\end{array}$ & 0 & 4 & 10 & 17 & 44 & 44 & 44 \\
$(0 \%)$ & $(9.09 \%)$ & $(22.72 \%)$ & $(38.63 \%)$ & $(100 \%)$ & $(100 \%)$ & $(100 \%)$ \\
\hline
\end{tabular}

Table-2: Radiological evidence of healing.

\begin{tabular}{|l|c|}
\hline $\mathbf{N}=\mathbf{4 4}$ & Number of subjects where it disappeared last radiologically \\
\hline Distal radius & 7 \\
\hline Distal ulna & 9 \\
\hline Distal femur & 27 \\
\hline Proximal tibia & 1 \\
\hline
\end{tabular}

Table-3: Mean change of pre- and post-treatment radiological score (by paired t-test).

\begin{tabular}{|l|c|c|c|c|c|c|c|c|c|}
\hline & $\mathbf{0 - 3}$ & $\mathbf{3 - 6}$ & $\mathbf{6 - 1 2}$ & $\mathbf{3 m - 6 m}$ & $\mathbf{6 m - 9 m}$ & $\begin{array}{c}\mathbf{9 m -} \\
\mathbf{1 2 m}\end{array}$ & $\begin{array}{c}\mathbf{0 - 6} \\
\text { weeks }\end{array}$ & $\begin{array}{c}\mathbf{0 - 1 2} \\
\text { weeks }\end{array}$ & $\mathbf{0 - 6 m}$ \\
\hline $\begin{array}{l}\text { Mean } \\
\text { change }\end{array}$ & 2 & 2.35 & 1.3 & 1.3 & 0 & 0 & 4.3 & 6.6 & 6.9 \\
\hline $\begin{array}{l}\text { Standard } \\
\text { deviation } \\
\text { change }\end{array}$ & 0.645 & 0.971 & 1.128 & 1.203 & - & - & 0.939 & 1.450 & 2.377 \\
\hline $\begin{array}{l}\text { Standard } \\
\text { error } \\
\text { mean }\end{array}$ & 0.097 & 0.146 & 0.170 & 0.181 & - & - & 0.142 & 0.219 & 0.358 \\
\hline P value & 0.000 & 0.000 & 0.000 & 0.000 & - & - & 0.000 & 0.000 & 0.000 \\
\hline
\end{tabular}


Original Research Article

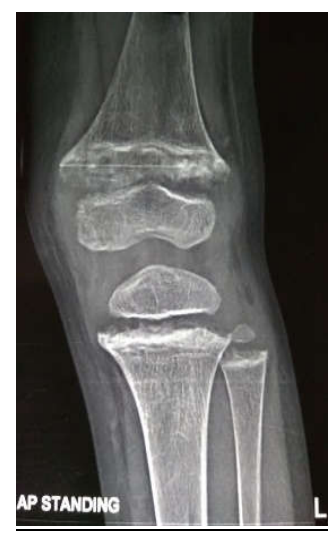

(A)

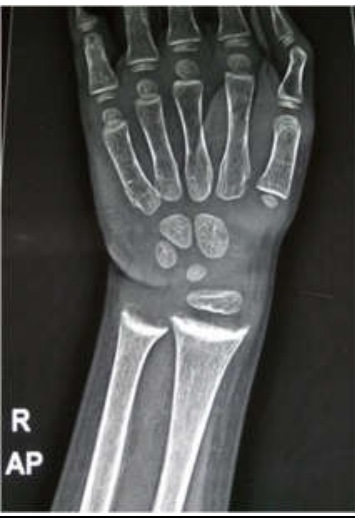

(B)

Figure 1: (a) Anteroposterior view of radiograph of the knee at initial presentation; score $3+3=6$.

(b) Anteroposterior view of radiograph of the wrist at initial presentation; score $2+2=4$. Total score $=10$.

Metaphyseal cupping, fraying, splaying and epiphyseal separation is evident

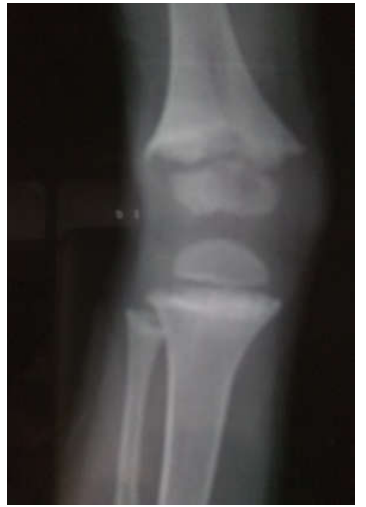

(A)

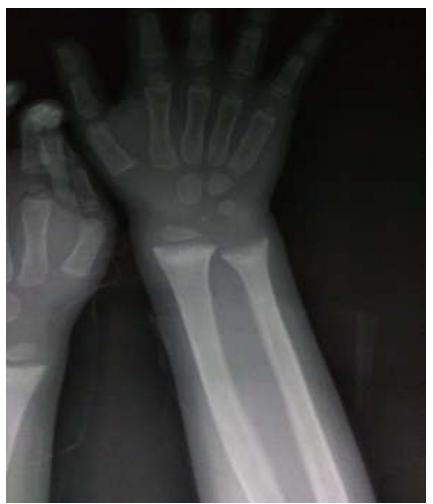

(B)

Figure 2: (a) Anteroposterior view radiograph of the knee at 3 weeks; score $3+2=5$.

(b)Anteroposterior view radiograph of the wrist at 3 weeks; score $2+2=4$.

Healing line of rickets is evident in figure $2 b$.

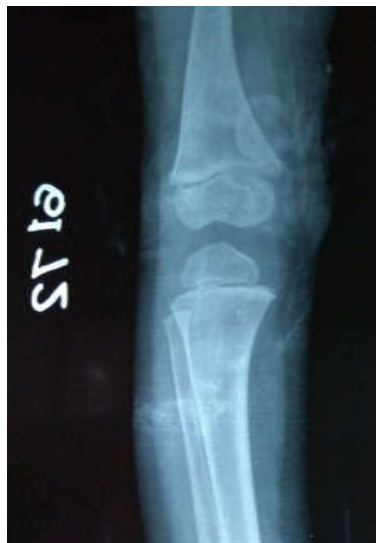

(A)

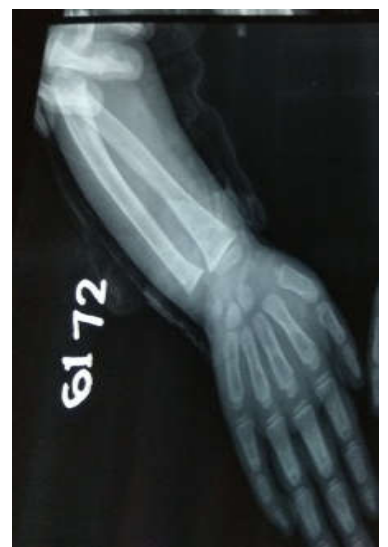

(B)

Figure 3: (a) Anteroposterior view radiograph of the knee at 6 weeks; score $2+0.5=2.5$.

(b) Anteroposterior view radiograph of the wrist at 6 weeks; score $1+0=1$. Total score $=3.5$ 


\section{Original Research Article}

Widened growth plate, irregularity of metaphyseal margin, but without concave cupping is evident in figure 3(b).

Partial lucency, smooth margin of metaphysis not visible in both condyle of femur in figure 3(a). whereas in tibia only one condyle involved.

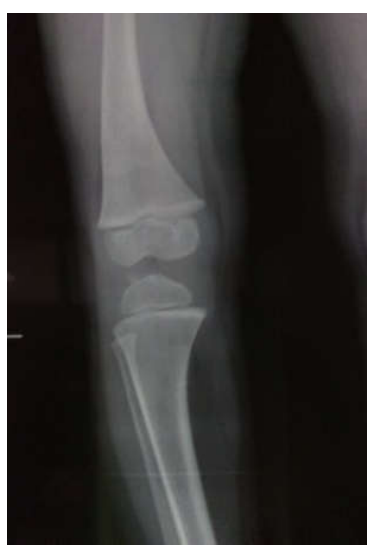

(A)

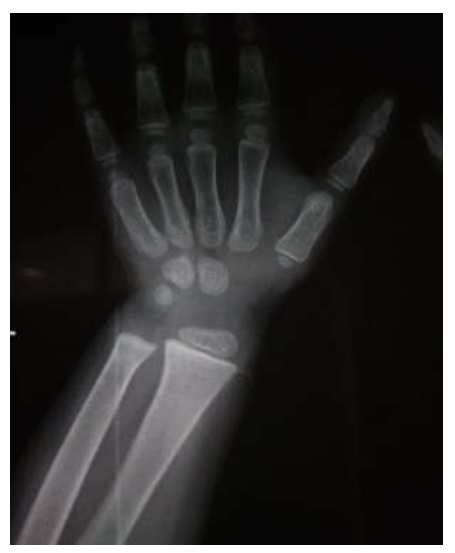

(B)

Figure 4: (a) anteroposterior view radiograph of the knee at 3 months; score $1+0=1$.

(b) Anteroposterior view radiograph of the wrist at 3 months; score $0+0=0$.

Total score $=1$.

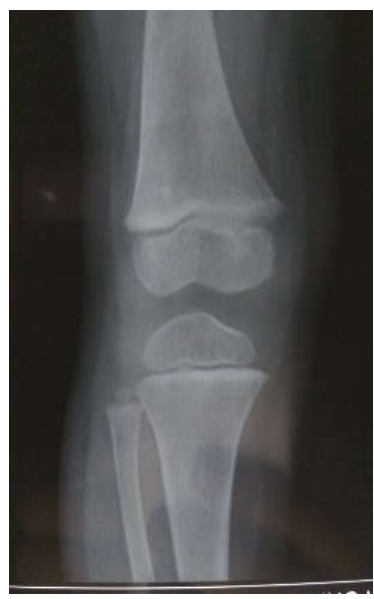

(A)

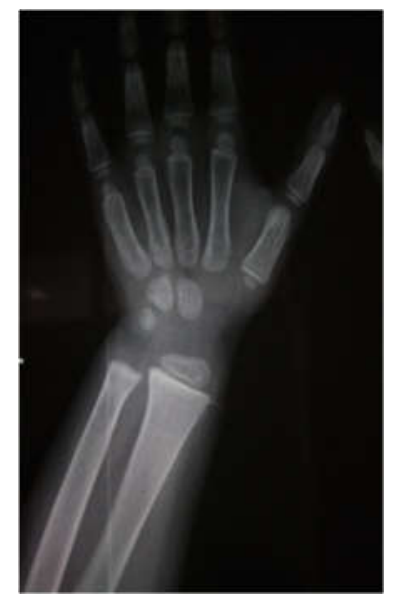

(B)

Figure 5 (a)Anteroposterior view radiograph of the knee at 6 months; score $0+0=0$.

(b)Anteroposterior view radiograph of the wrist at 6 months; score $0+0=0$.

Complete healing of metaphysis and epiphysis of radius, ulna, femur, tibia evident.

Total score $=0$.

The first response of resolution was the healing line of rickets found in $83 \%$ cases by 3 weeks and in $100 \%$ cases by 6 weeks. The order of resolution was as follows: first the cupping disappeared followed by fraying and finally splaying. It was found that initial radiographic changes were present in the distal ulna in $100 \%$ of cases followed by the distal radius in $93 \%$ of cases. Distal femur was the last to heal in about 27 out of 44 cases. (Table 2) However, in all the 8 cases having an initial radiological score of 10 , the distal femur was the last to heal. It was also noted that, when the radiological score took more than 12 weeks to resolve, the distal femur was the last to heal in the majority of the cases. None of the subjects had initial score zero. After starting treatment, 4 subjects out of 44 had zero score whereas at 6 weeks, 3 month 10 subjects and 17 subjects had score zero respectively. At 6 month all subject had score zero. Distal femur last to heal in majority of patient after Stoss therapy. 


\section{Original Research Article}

All of the study subjects achieve 0 radiological score by 6 months. All the changes of radiological scores during the follow up were found to be statistically significant by paired $t$ test. 8 subjects were found to have an initial radiological score of 10 .

\section{Discussion}

A female preponderance in nutritional rickets may be due to discrimination against the female gender in India. High atmospheric pollution in Delhi filtering ultraviolet rays may be another factor. Inadequate sunlight exposure at presentation and breast feeding for more than 6 months were found to increase the incidence, severity and recovery time in nutritional rickets. Researchers in Greater New Haven [9] conducted a study on 43 children with nutritional rickets, and they also concluded that inadequate sunlight exposure and prolonged breast feeding were the predominant risk factors for rickets.

Thus, it is recommended that awareness needs to be generated regarding the role of adequate sunlight exposure (30 min per week) [10-17] and discontinuance of breast feeding beyond 6 months [18-23] as a preventive strategy for rickets.

The first radiological sign of response is the appearance of the healing line of rickets, which is the appearance of the radio-opaque line in the epiphysis signifying that mineralization of the provisional zone of calcification. The first response of resolution found in $100 \%$ cases by 6 weeks. The order of resolution was as follows: first the cupping disappeared followed by fraying and finally splaying. It was found that initial radiographic changes were present in the distal ulna in $100 \%$ of cases followed by the distal radius, distal femur and proximal tibia in $88.6 \%, 95.5 \%, 90.9 \%$ of cases respectively.

It was found that radiological sign of nutritional rickets was decreased with time with Stoss therapy and were absent in $100 \%$ of cases by 6 months. Like in distal ulna $100 \%$ of cases have radiological sign of rickets and by 3 weeks, 6 weeks, 3 months, 6 months only $65.9 \%$, $31.8 \%, 18.2 \%$ and $0 \%$ of cases respectively had radiological sign of rickets. This shows that initial radiological score can be used for monitoring of healing of rickets.

None of the subject had any radiological sign of nutritional rickets once radiological resolution completed during follow up. It was seen that although distal ulna is more specific as an indicator of completion of radiological healing, but distal femur is more specific when the initial score was 10 and when complete radiological healing was not achieved by 12 weeks. Time taken for radiological score to resolve completely was found positively with initial radiological score. It was also observed that the initial radiological score could explain the variability in the time needed for radiological score to resolve completely in $93 \%$ cases. All the changes in mean radiological scores during the follow-up were found to be statistically significant by paired t-test.

This signifies that radiography at every follow up visit will show significant changes, which can be aptly scored and used for monitoring the outcome. But to avoid multiple radiological exposures during follow-up, it is suggested to repeat radiography once at 6 weeks to look for white lines of rickets and at the expected time of resolution predicated by the formula. This decrease not only the number of radiological exposures but also the cost of treatment (which is especially important in underdeveloped and developing countries where rickets is endemic and a free radiographic facility is not available at all the health centres), and eventually improves the compliance of the patient.

It has been observed that children are more sensitive to the carcinogenic effects of ionizing radiation than adults. Moreover, children have a longer life expectancy than adults, thus increasing the chances of expressing radiation damage [8]. Hence, children are to receive minimum radiation exposure whenever possible.

Studies conducted in VMMC, New Delhi [8] in 2013 on 176 children with features of nutritional rickets revealed similar results regarding this formula based on initial radiological score as that of the present study.

Studies conducted at UCMS hospital, New Delhi [5] in 2009 on 40 females and 11 males between 10-13 years with features of rickets treated by a single large intramuscular dose of Vitamin-D (600 000 IU) along with oral calcium and supplementary Vitamin-D as well as advice on diet and sunlight exposure revealed similar results regarding radiological improvement with treatment in rickets as that of the present study. It was observed that all patients had rachitic changes on radiographs in some but not all bones $100 \%$ in the ulna, $45 \%$ in the radius, $37 \%$ in the upper tibia, $37 \%$ in the lower fibula and $22 \%$ in the lower tibia. For radiological resolution it was 5 (range 2-6) months, with the lower end of ulna being last to resolve. 


\section{Original Research Article}

Limitations of the present study- The present study had few drawbacks, absence of any control group, as well as smaller number of participants in the study. However, considering the availability of only a few research data in literature at present on radiological resolution of nutritional rickets with mega dose of vitamin D as per Stoss Therapy regimen, the results of the present study can be considered significant.

\section{Conclusion}

Stoss therapy (mega dose of vitamin D) is very effective and safe regime for the treatment of rickets. The 10 points Thacher's scoring method for assessing the severity of rickets shows promise as a useful tool for researchers and clinicians.

In view of reducing the cost of therapy and minimizing the radiation exposure, only antero-posterior radiographs of the wrist are recommended for both diagnosis and follow up in rickets as distal ulna was found to have characteristic radiographic signs of rickets in $100 \%$ of cases at initial presentation and was also last to heal in a maximum number of cases. However, when initial score is 10 , the distal femur used as better indicator for follow up.

\section{What this study adds to existing knowledge?}

The distal femur is a more specific indicator in radiologically severe rickets and when resolution is delayed. Thacher's grading is very useful for monitoring response to treatment in children with rickets.

\section{Author's contributions}

Dr. Manoj Kumar, Dr. Mozammil Pheroz and Dr. Vikas Gupta conceived and designed the analysis. Dr. Manoj Kumar and Dr. Vikash Kumar Pandey collected the data. Dr. Ankit Jain and Dr. Vasu Sharma contributed data and analysis tools. Dr. Mozammil Pheroz and Dr. Manoj Kumar performed the analysis and wrote the paper.

Funding - No funding from any source.

Conflict of interest -none.

Permission from IRB- Yes

\section{References}

1. Shore R, Chesney RW. Rickets: Part i. Pediatric Radiol. 2013; 43 (2):140-151. doi: https://doi.org/10. 1007/s00247 -012-2532-x.
2. Bhattacharyya AK. Nutritional rickets in the tropics. World Rev Nutr Diet. 1992:67:140-197.

3. Muhe L, Lulseged S, Mason KE, Simoes EA. Casecontrol study of the role of nutritional rickets in the risk of developing pneumonia in Ethiopian children. The Lancet. 1997;349(9068):1801-1804. doi: https://doi.org/ 10.1016/S0140-6736(96)12098-5.

4. Thacher TD, Fischer PR, Pettifor JM, Lawson JO, Isichei $\mathrm{CO}$, Reading JC, Chan GM. A comparison of calcium, vitamin $\mathrm{D}$, or both for nutritional rickets in Nigerian children. New Eng J Med. 1999;341(8):563568. doi: 10.1056/NEJM199908193410803.

5. Agarwal A, Gulati D. Early adolescent nutritional rickets. J Orthopaed Sur. 2009;17(3):340-345. doi: https://doi.org /10.1177/230949900901700320.

6. Thacher TD, Fischer PR, Pettifor JM, Lawson JO, Manaster BJ, Reading JC. Radiographic scoring method for the assessment of the severity of nutritional rickets. $J$ Trop Pediat. 2000;46(3):132-139. doi: 10.1093/ tropej/ 46.3.132.

7. Woolf AD, Pfleger B. Burden of major musculoskeletal conditions. Bull World Health Organ. 2003;81(9):646-656. Epub 2003 Nov 14.

8. Chatterjee D, Gupta V, Sharma V, Sinha B, Samanta S. A reliable and cost-effective approach for radiographic monitoring in nutritional rickets. $\mathrm{Br} \mathrm{J}$ Radiol. 2014;87(1036):20130648. doi: 10.1259/bjr. 20130648.

9. DeLucia MC, Mitnick ME, Carpenter TO. Nutritional rickets with normal circulating 25-hydroxyvitamin D: a call for reexamining the role of dietary calcium intake in North American infants. $J$ Clinic Endocrinol Metabol. 2003;88 (8):3539-3545. doi: https://doi.org /10.1210/jc.2002-021935.

10. Hillman LS. Mineral and vitamin D adequacy in infants fed human milk or formula between 6 and 12 months of age. J Pediatr. 1990;117(2 Pt 2):S134-S142. doi: 10.1016/s0022-3476(05)80011-3.

11. Glerup H, Mikkelsen K, Poulsen L, Hass E, Overbeck S, Thomsen $\mathrm{J}$, et al. Commonly recommended daily intake of vitamin D is not sufficient if sunlight exposure is limited. J Int Med. 2000;247 (2):260-268. Doi :https://doi.org/10.1046/j. 1365-2796. 2000.00595.x. 


\section{Original Research Article}

12. Pettifor JM. Vitamin D \&/or calcium deficiency rickets in infants \& children: a global perspective. Indian J Med Res. 2008;127(3):245-249.

13. Holick MF. Vitamin D deficiency. N Engl J Med. 2007;357(3):266-281. doi: 10.1056/NEJMra070553.

14. Jones $\mathrm{G}$, Dwyer $\mathrm{T}$. Bone mass in prepubertal children: gender differences and the role of physical activity and sunlight exposure. J Clin Endocrinol Metab. 1998;83(12):4274-4279. doi: 10.1210/jcem. 83.12.5353.

15. Reid IR, Gallagher DJ, Bosworth J. Prophylaxis against vitamin $\mathrm{D}$ deficiency in the elderly by regular sunlight exposure. Age Ageing. 1986;15(1):35-40. doi: 10.1093/ageing/15.1.35.

16. Sato $\mathrm{Y}$, Iwamoto $\mathrm{J}$, Kanoko $\mathrm{T}$, Satoh $\mathrm{K}$. Amelioration of osteoporosis and hypovitaminosis D by sunlight exposure in hospitalized, elderly women with Alzheimer's disease: a randomized controlled trial. J Bone Miner Res. 2005;20(8):1327-1333. doi: 10.1359/ JBMR.050402.

17. Kumar A, Pheroz M, Chopra RK, Tungoe B, Kumar $\mathrm{N}$, Aggarwal $\mathrm{Y}$, et al. Evaluation of relationship between bone mineral density and fragility fracture in perimenopausal women between 40-58 years of age: a hospital based prospective observational study. Int J Res Orthopaed. 2019;5(4):605-611. doi: http://dx.doi.org/ 10.18203/issn.2455-4510.IntJResOrthop20192284.
18. Gartner LM, Greer FR; Section on Breastfeeding and Committee on Nutrition. American Academy of Pediatrics. Prevention of rickets and vitamin D deficiency: new guidelines for vitamin $\mathrm{D}$ intake. Pediatr. 2003;111(4 Pt 1):908-910. doi: 10.1542/ peds.111.4.908.

19. Bhowmick SK, Johnson KR, Rettig KR. Rickets caused by vitamin $\mathrm{D}$ deficiency in breast-fed infants in the southern United States. Am J Dis Child. 1991;145(2):127-130. doi: 10.1001/archpedi.1991. 0216 0020017006.

20. Eugster EA, Sane KS, Brown DM. Minnesota rickets. Need for a policy change to support vitamin D supplementation. Minn Med. 1996;79(8):29-32.

21. Edidin DV, Levitsky LL, Schey W, Dumbovic N, Campos A. Resurgence of nutritional rickets associated with breast-feeding and special dietary practices. Pediatr. 1980;65(2):232-235.

22. Tomashek KM1, Nesby S, Scanlon KS, Cogswell ME, Powell KE, Parashar UD, et al. Nutritional rickets in Georgia. Pediatr. 2001;107(4):e45. doi: https://doi. org/ 10.1542/peds.107.4.e45.

23. Mughal MZ, Salama H, Greenaway T, Laing I, Mawer EB. Florid rickets associated with prolonged breast feeding without vitamin D supplementation. Bmj. 1999;318(7175):39-40. doi: 10.1136/bmj.318.7175.39.

\section{How to cite this article?}

Kumar M., Pheroz M., Gupta V., Pandey V.K., Jain A., Sharma V., Radiological resolution of nutritional rickets with mega dose of vitamin D - "Stoss Therapy": a hospital-based study. Surgical Update: Int J surg Orthopedics.2019;5(4):300-307.doi:10.17511/ijoso.2019.i04.11 